\title{
Avaliação dos movimentos em recém-nascidos prematuros internados em uma unidade de cuidados intermediários neonatal
}

\author{
Movement assessment in premature newborns admitted to a neonatal intermediate care unit \\ Evaluación de movimientos en recién nacidos prematuros ingresados en unidad de cuidados \\ intermedios neonatales
}

Recebido: 03/11/2021 | Revisado: 10/11/2021 | Aceito: 16/11/2021 | Publicado: 26/11/2021

Thaís Dutra Rodrigues

ORCID: https://orcid.org/0000-0002-1716-3434

Faculdade Independente do Nordeste, Brasil

E-mail: tai.d.r@hotmail.com

Mariane Soares Barros

ORCID: https://orcid.org/0000-0002-0441-4660

Faculdade Independente do Nordeste, Brasil E-mail: mariane0soares@gmail.com

Laisla Pires Dutra

ORCID: https://orcid.org/0000-0003-4111-7101

Faculdade Independente do Nordeste, Brasil

E-mail: laysla19@hotmail.com

\begin{abstract}
Resumo
Objetivo: Avaliar os movimentos em recém-nascidos prematuros internados em uma unidade de cuidados intermediários neonatal, segundo a General Movements Assessment (GMA). Métodos: Trata-se de um estudo descritivo, exploratório de abordagem quantitativa e delineamento transversal, realizado em um Hospital MaternoInfantil no interior da Bahia, no período de julho a setembro de 2021, composto por uma amostra de 20 recémnascidos pré-termos. Os instrumentos utilizados para coleta de dados da pesquisa foram: formulário do prontuário dos recém-nascidos pré-termos e a escala de GMA. Resultados: A população do estudo apresentou idade gestacional corrigida entre 33 e 39 semanas correspondendo a fase dos Writhing Movements, foi possível identificar que 65\% apresentaram movimentação anormal, sendo 55\% preditivo para paralisia cerebral espástica. Conclusão: $\mathrm{O}$ Profissional fisioterapeuta além de realizar intervenção neurosensoriomotora em recém-nascidos de alto risco para o desenvolvimento infantil, realiza avaliação a fim de detectar sinais clínicos para atrasos de desenvolvimento motor. Toda intervenção tem a finalidade de adequar o tônus muscular, diminuir a dor, estimular as sinergias musculares e facilitar o desenvolvimento neurosensóriomotor, percebeu-se no estudo que o instrumento de avaliação GMA, é um facilitador confiável, desde idades gestacionais bem precoces.
\end{abstract}

Palavras-chave: Recém-nascido prematuro; Unidade de Terapia Intensiva Neonatal; Fisioterapia.

\begin{abstract}
:
Objective: To evaluate the movements in premature newborns hospitalized in a neonatal intermediate care unit, according to the General Movements Assessment (GMA). Methods: This is a descriptive, exploratory study with a quantitative approach and cross-sectional design, conducted in a Maternal and Infant Hospital in the interior of Bahia, from July to September 2021, consisting of a sample of 20 preterm newborns. The instruments used for data collection of the research were: form from the medical record of the preterm newborns and the GMA scale. Results: The study population presented corrected gestational age between 33 and 39 weeks corresponding to the Writhing Movements phase, it was possible to identify that $65 \%$ presented abnormal movement, being $55 \%$ predictive for spastic cerebral palsy. Conclusion: The professional physiotherapist, besides performing neurosensory-motor intervention in newborns at high risk for infant development, performs an evaluation in order to detect clinical signs of motor development delays. Every intervention is intended to adjust muscle tone, reduce pain, stimulate muscle synergies and facilitate neurosensory-motor development, it was realized in the study that the GMA assessment instrument, is a reliable facilitator, since very early gestational ages.
\end{abstract}

Keywords: Premature newborn; Neonatal Intensive Care Unit; Physical therapy.

\section{Resumen:}

Objetivo: Evaluar los moviminetos en recién nacidos prematuro internado en la unidad de cuidados intermediários neonatal, de acuerdo con la General Movements Assessment (GMA). Métodos: se trata de un estúdio descriptivo, escrutínio de abordaje cuantitativa y delineación transversal, realizado em un Hospital Materno-infantil en el interior 
de la Bahia, en el período de julio hasta septiempre del año 2021, compuesto por uma mostra de 20 (veinte) recién nacidos prematuros. Los instrumentos utilizados para la colecta de datos del estudio fueron: formulário del historial médico de los recién nacidos y la escala GMA. Resultados: La población del estúdio ha presentado edad gestacional corregida entre 33 y 39 semanas equivalente a la fase de los Writhing Movements, fue posible identificar que $65 \%$ ha presentado movimentación anormal, siendo 55\% con predisposición para parálisis cerebral espástico. Conclusión: El profissional de fisioterapia realiza intervención neurosensoriomotor en recién nacidos de elevado riesgo para el desarrollo infantil, realiza la evaluación con la finalidade de detectar señales clínicos para retrasos de desenrolo motor. La intervención tiene la finalidade de adecuar el tono muscular, disminuir la dolor, impulsar las sinergias musculares y facilitar el desarrollo neurosensoriomotor, comprendió en el estúdio que el instrumento de evaluación GMA, es un facilitador confiable, desde edades gestacionales precoz.

Palabras clave: Recién nacidos prematuros; Unidad de Cuidados Intermediários Neonatal; Fisioterapia.

\section{Introdução}

O Ministério da Saúde (MS) destaca que, no Brasil, cerca de 10\% dos partos são realizados precocemente (menor que 37 semanas de idade gestacional), caracterizados como prematuros (Brasil, 2016). Segundo a Organização Pan-Americana da Saúde (OPAS) o percentual de crianças que nascem mundialmente com um fator de risco, não consegue sobreviver ao primeiro mês após o nascimento. Em 2017, dois milhões e meio das mortes de bebês no mundo foram por causas evitáveis e aproximadamente 2/3 delas foram de prematuros. Contudo, os recém-nascidos prematuros que sobrevivem apresentam risco e complicações para o desenvolvimento (Opas, 2018).

$\mathrm{Na}$ fase intrauterina o feto perpassa pela formação e pelo desenvolvimento de diversos sistemas que compõem o ser humano. Melo e Belém (2020) afirmam que a transferência nutricional indispensável para o ganho de peso, o crescimento intrauterino e o desenvolvimento do sistema nervoso do feto ocorrem no terceiro trimestre da gestação, período gestacional em que normalmente há interrupção quando se trata de parto prematuro. Dessa forma, o bebê pré-termo possivelmente terá atraso no crescimento, no desenvolvimento neuropsicomotor, bem como, no cognitivo, além de, em sua maioria, nascerem com baixo peso associado à prematuridade. Esses lactentes apresentam índices elevados de morbimortalidade, necessitam de internações prolongadas na Unidade de Terapia Intensiva Neonatal (UTIN) e consequentemente estão mais predispostos a atraso motor (Rodrigues, 2017).

A UTIN apesar de ser imprescindível para a sobrevivência do prematuro, pode colaborar com as alterações no desenvolvimento motor do neonato, visto que este apresenta imaturidade do sistema neurofisiológico (Salvagni; Gerzson; Almeida, 2020). Ainda segundo os autores supracitados, o bebê fica exposto a estímulos negativos durante o internamento como procedimentos dolorosos, excesso de estimulação, luminosidade e ruídos, sendo fatores que favorecem o atraso no desenvolvimento motor.

Apesar do padrão de movimento em recém-nascidos ser ainda imaturo, é possível observar uma movimentação atípica em recém-nascidos pré-termos (RNPT) quando comparados aos recém-nascidos termos (RNT). Desse modo, Salvagni e colaboradores (2020) afirmam a importância de identificar esses atrasos precocemente, ainda na UTIN, uma vez que o diagnóstico precoce é norteador para a intervenção. A literatura ressalta que a utilização de instrumentos de avaliação é importante para identificar alteração no movimento de lactentes prematuros e podem ser facilmente utilizadas na unidade intensiva.

Dentre os instrumentos de avaliação utilizados precocemente, encontra-se a General Movements Assessment (GMA), utilizada para avaliar a integridade do sistema nervoso central por meio dos movimentos gerais realizados pelos bebês. Einspieler et al. (2016a; 2016b), afirmam que essa ferramenta é importante para detectar predisposição de risco de movimentos anormais em recém-nascidos termos e pré-termos, sendo que em prematuros é possível identificar além do padrão anormal de movimento atraso no desenvolvimento cognitivo. A associação da GMA com exames de imagens e neurológico podem 
prognosticar a paralisia cerebral (PC) em bebês de risco de acordo com (Van Iersel et al., 2016; Einspieler et al., 2016a; 2016b).

Diante dos contextos apresentados, é possível salientar a importância em estudar as alterações do padrão de movimento em lactentes prematuros, uma vez que é através de pesquisas científicas que os profissionais da área da saúde aperfeiçoam seus conhecimentos e técnicas para intervenção. Desse modo, o objetivo do atual estudo é avaliar os movimentos em recém-nascidos prematuros internados numa unidade de cuidado intermediário neonatal, segundo a General Movements Assessment.

\section{Metodologia}

Trata-se de um estudo descritivo e exploratório, visto que a pesquisa visa retratar as características específicas de um grupo da população, bem como, correlacionar variáveis, além de possibilitar maior domínio sobre o problema, tornando-o mais explícito (Gil, 2017). O estudo é de abordagem quantitativa, que é aquela em que os resultados são apresentados em formas numéricas, quantificando-os, sendo possível desenvolver a coleta de dados por meio de entrevistas ou de modo observacional (Gil, 2010) e de delineamento transversal, ou seja, um estudo cujas análises são observadas em um mesmo período histórico (Bordalo, 2006).

A pesquisa foi aprovada pelo Comitê de Ética e Pesquisa (CEP) por meio do parecer nº4.817.091 e somente após a aprovação foi iniciada em um hospital materno-infantil, escolhido pelo critério de conveniência, localizado no interior da Bahia, Brasil. A amostra foi não probabilística por conveniência, constituída por 20 prematuros internados na unidade de cuidados intermediários do hospital maternidade e 17 genitoras que preencheram o formulário construído pelas pesquisadoras do estudo. Os dados foram coletados entre os meses de julho a setembro de 2021.

Participaram do estudo todos os prematuros internados na semi-intensiva, independentes da idade gestacional ao nascimento, nascidos por parto (normal, cesárea ou fórceps), de ambos os sexos, que tiveram ou não intercorrências neonatais, e que estavam em ventilação espontânea ou ar ambiente durante o período da coleta de dados. Foram excluídos do estudo prematuros que apresentaram sinais de desconforto respiratório no momento da coleta de dados, que estiveram com alteração nos SSVV (FC, FR, SpO2, PA, $\mathrm{T}^{\circ}$ ) apresentados no monitor da unidade ou em uso de medicação vasodilatadoras ou sedativos.

Os instrumentos para coleta de dados foram duas fichas, sendo a primeira um formulário para a coleta de dados do prontuário dos recém-nascidos prematuros participantes do estudo, construído pelas pesquisadoras, contendo dois blocos, sendo eles: Bloco A - informações sobre a História Obstétrica (Duração da Gestação; Tipo de Gravidez; Tipo de Parto; $\mathrm{N}^{\circ}$ de Consultas Pré-natal e Patologias Durante a Gestação) e no Bloco B - Caracterização do RN que inclui os seguintes dados (Sexo; Cor/etnia; Índice de Apgar do $1^{\circ}$ e $5^{\circ}$ minuto de vida; Peso ao Nascer; Idade Gestacional; Data de Nascimento; Idade Cronológica; Idade Corrigida; Suporte Ventilatório; Tempo de Internamento na UTIN; Tempo de Internamento na Semiintensiva, bem como, Diagnóstico ao Nascer); A outra ficha é intitulada por "Método de Prechtl na Avaliação do Movimento Geral - Trajetória de Desenvolvimento Individual” a qual contém sete itens a serem observados no Rn (N: Movimentos Gerais normais para a idade; FMs: movimentos inquietos; H: hipocinesia - sem movimentos gerais durante a gravação; PR: repertório pobre de movimentos gerais; Ch: movimentos gerais caóticos; CS: movimentos gerais sincronizados com restrições; AF: movimentos inquietos anormais; F-: ausência de movimentos inquietos).

A coleta de dados aconteceu em duas etapas. A primeira, realizada por meio da seleção das participantes do estudo, segundo os critérios de inclusão do estudo, após a identificação destas, foi apresentado as mesmas o Termo de Consentimento Livre e Esclarecido (TCLE) e o Termo de autorização de imagem onde foi explicado as genitoras todas as etapas da pesquisa, bem como seus objetivos, elucidando aos responsáveis a autonomia de sair do estudo durante qualquer fase e explicando de forma clara o caráter voluntário e anônimo da pesquisa. Concedida a autorização através da assinatura do TCLE e do Termo 
de Autorização de Imagem, na segunda etapa foram feitas as filmagens dos bebês pelas pesquisadoras do estudo numa média de tempo de 3 minutos, com o auxílio de smartphone da Apple, Iphone 7 e 8 para análise detalhada dos movimentos gerais dos prematuros, por fim, foi realizada a avaliação observacional das filmagens e o preenchimento dos dados na ficha da General Movements Assessment (GMA), como já descritos no item de instrumento de coleta deste projeto.

Para organização dos dados quantitativos, foi construída uma planilha eletrônica, através do programa Excel. A análise ocorreu de forma descritiva dos dados, verificando a média, desvio-padrão, porcentagem, frequência absoluta e relativa.

\section{Resultados e Discussão}

O estudo foi inicialmente constituído por 23 RNPT internados na unidade de cuidados intermediários neonatal e 20 genitoras. Após a aplicação dos critérios de inclusão e exclusão foram selecionados 20 RNPT e 17 genitoras aos quais foram aplicados o formulário construido pelas pesquisadoras do estudo com dados obstetricos, o TCLE e o termo de autorização de imagem, devido a segurança da aplicação do GMA, para posterior análise.

A Tabela 1 apresenta a caracterização dos dados obstétricos, cujo tipo de gravidez da maioria das participantes foi única $(\mathrm{n}=15)$, o $\mathrm{n}^{\circ}$ de consultas realizadas variou entre 6 a 7 consultas. A maioria das participantes $(\mathrm{n}=9)$ apresentaram patologias obstétricas. Em relação à duração da gestação, 59\% $(\mathrm{n}=10)$ foi pré-termo moderado e tipo de parto predominantemente abdominal $(\mathrm{n}=13)$, conforme segue. 
Tabela 1. Caracterização dos dados obstétricos das genitoras participantes do estudo. Vitória da Conquista, Bahia, Brasil. 2021.

\begin{tabular}{|c|c|c|c|c|}
\hline Variáveis & Frequência & Porcentagem & Porcentagem Válida & Porcentagem Acumulativa \\
\hline \multicolumn{5}{|c|}{ Variável 1: Tipo de gravidez } \\
\hline ÚNICA & \multirow{4}{*}{$\begin{array}{c}15 \\
1 \\
1 \\
17 \\
\end{array}$} & \multirow{4}{*}{$\begin{array}{c}88 \% \\
6 \% \\
6 \% \\
100 \% \\
\end{array}$} & \multirow{4}{*}{$\begin{array}{c}88 \% \\
6 \% \\
6 \% \\
100 \% \\
\end{array}$} & \multirow{4}{*}{$\begin{array}{l}88 \% \\
94 \% \\
100 \%\end{array}$} \\
\hline GEMELAR & & & & \\
\hline TRIGEMELAR & & & & \\
\hline TOTAL & & & & \\
\hline \multicolumn{5}{|c|}{ Variável 2: $\mathbf{N}^{0}$ de Consulta } \\
\hline 1- Não realizou & \multirow{5}{*}{$\begin{array}{c}0 \\
6 \\
6 \\
5 \\
17 \\
\end{array}$} & \multirow{5}{*}{$\begin{array}{c}0 \% \\
35 \% \\
35 \% \\
29 \% \\
100 \% \\
\end{array}$} & \multirow{5}{*}{$\begin{array}{c}0 \% \\
35 \% \\
35 \% \\
29 \% \\
100 \% \\
\end{array}$} & \multirow{5}{*}{$\begin{array}{c}0 \% \\
35 \% \\
71 \% \\
100 \%\end{array}$} \\
\hline $2-\leq 6$ & & & & \\
\hline $3-\geq 7$ & & & & \\
\hline 4- Não sabe Informar & & & & \\
\hline TOTAL & & & & \\
\hline \multicolumn{5}{|c|}{ Variável 3: Presença de patologia obstétricas } \\
\hline SIM & \multirow{3}{*}{$\begin{array}{c}9 \\
8 \\
17 \\
\end{array}$} & \multirow{3}{*}{$\begin{array}{r}53 \% \\
47 \% \\
100 \% \\
\end{array}$} & \multirow{3}{*}{$\begin{array}{r}53 \% \\
47 \% \\
100 \% \\
\end{array}$} & \multirow{3}{*}{$\begin{array}{l}53 \% \\
100 \%\end{array}$} \\
\hline NÃO & & & & \\
\hline TOTAL & & & & \\
\hline \multicolumn{5}{|c|}{ Variável 4: Duração da Gestação } \\
\hline Pré termo limítrofe & \multirow{4}{*}{$\begin{array}{c}6 \\
10 \\
1 \\
17 \\
\end{array}$} & \multirow{4}{*}{$\begin{array}{c}35 \% \\
59 \% \\
6 \% \\
100 \% \\
\end{array}$} & \multirow{4}{*}{$\begin{array}{c}35 \% \\
59 \% \\
6 \% \\
100 \% \\
\end{array}$} & \multirow{4}{*}{$\begin{array}{l}35 \% \\
94 \% \\
100 \%\end{array}$} \\
\hline Pré termo moderado & & & & \\
\hline Pré termo extremo & & & & \\
\hline TOTAL & & & & \\
\hline \multicolumn{5}{|c|}{ Variável 5: Tipo de parto } \\
\hline 1- Vaginal & 4 & $24 \%$ & $24 \%$ & $24 \%$ \\
\hline 2- Abdominal & 13 & $76 \%$ & $76 \%$ & $100 \%$ \\
\hline TOTAL & 17 & $100 \%$ & $100 \%$ & \\
\hline
\end{tabular}

Fonte: Dados da pesquisa.

A Organização Mundial da Saúde (OMS) define o recém-nascido prematuro como aquele que nasce com idade gestacional inferior a 37 semanas. A prematuridade é classificada de acordo com a idade gestacional (IG) em prematuridade limítrofe (IG de $35^{\mathrm{a}}$ e $36^{\mathrm{a}}$ semana e 6 dias), moderada (IG de $31^{\mathrm{a}}$ a $34^{\mathrm{a}}$ semanas e 6 dias) e extrema (IG menor ou igual a 30 semanas) conforme a OMS (2018). Segundo Lopes (2017), o RN pode ser ainda classificado de acordo com o seu peso em: extremo baixo peso, aqueles que nascem pesando menos $1000 \mathrm{~g}$, muito baixo peso inferior a $1500 \mathrm{~g}$ e é considerado baixo peso o bebê que nasce com menos de $2.500 \mathrm{~g}$. Sendo que, a prematuridade extrema associada ao baixo peso oferece a esse RN menor sobrevida.

Os fatores de risco para o parto prematuro, segundo Penha et al. (2019) incluem: fatores sociodemográficos como idade materna menor do que 20 anos ou maior do que 35 anos, baixa escolaridade, estado civil solteira, a ocupação, uso de drogas lícitas e/ou ilícitas, má nutrição gestacional, parto cesáreo, gravidez gemelar, antecedente de parto prematuro, ausência do pré natal ou número de consultas inadequadas, condições clínicas pré existentes, como hipertensão arterial sistêmica, diabetes mellitus e doenças cardiovasculares ou condições adquiridas durante o período gestacional, como pré-eclâmpsia, eclâmpsia, diabetes mellitus gestacional e infecção do trato urinário. Berger e colaboradores (2016) também citam como 
fatores de risco, o baixo nível socioeconômico, estresse, descolamento prévio de placenta, sangramentos, malformações fetais e crescimento intrauterino restrito.

De acordo com os dados coletados pelo formulário durante a pesquisa e apresentados na tabela 1 do atual estudo, a duração da gestação variou de 27 a 36 semanas de idade gestacional elucidando assim a prematuridade, de acordo com a OMS (2018). As genitoras apresentaram como fator de risco para a prematuridade gravidez gemelar, presença de patologias obstétricas, como DMG (Diabetes Mellitus Gestacional), DHEG (Doença Hipertensiva Exclusiva da Gestacão), pré-eclampsia, síndrome de HELLP, sífilis, CIUR (Crescimento Intrauterino Reduzido) e parto cesáreo corroborando com Penha et al. (2019) e Berger et al. (2016).

A Tabela 2 apresenta a caracterização dos recém-nascidos pré-termos, onde a maioria dos participantes foi do sexo feminino ( $\mathrm{n}=12)$, o apgar foi maior ou igual a $7(\mathrm{n}=16)$ no $1^{\circ}$ minuto e maior ou igual a $7(\mathrm{n}=18)$ também no $5^{\circ}$ minuto de vida. A maioria dos bebês nasceram com muito baixo peso $(n=12)$. Em relação a admissão, 50\% $(n=10)$ foram admitidos na UTIN e 85\% ( $\mathrm{n}=17)$ dos RNs necessitaram de suporte ventilatório, conforme segue.

Tabela 2. Caracterização dos recém-nascidos pré-termos participantes do estudo. Vitória da Conquista, Bahia, Brasil. 2021.

\begin{tabular}{|c|c|c|c|c|}
\hline Variáveis & Frequência & Porcentagem & Porcentagem Válida & $\begin{array}{l}\text { Porcentagem } \\
\text { Acumulativa }\end{array}$ \\
\hline \multicolumn{5}{|l|}{ Variável 1: Sexo } \\
\hline Feminino & 12 & $60 \%$ & $60 \%$ & $60 \%$ \\
\hline Masculino & 8 & $40 \%$ & $40 \%$ & $100 \%$ \\
\hline TOTAL & 20 & $100 \%$ & $100 \%$ & \\
\hline \multicolumn{5}{|l|}{ Variável 2: Apgar 1' } \\
\hline$<7$ & 4 & $20 \%$ & $20 \%$ & $20 \%$ \\
\hline$\geq 7$ & 16 & $80 \%$ & $80 \%$ & $100 \%$ \\
\hline TOTAL & 20 & $100 \%$ & $100 \%$ & \\
\hline \multicolumn{5}{|l|}{ Variável 3: Apgar 5' } \\
\hline$<7$ & 2 & $10 \%$ & $10 \%$ & $10 \%$ \\
\hline$\geq 7$ & 18 & $90 \%$ & $90 \%$ & $100 \%$ \\
\hline TOTAL & 20 & $100 \%$ & $100 \%$ & \\
\hline \multicolumn{5}{|l|}{ Variável 4: Peso } \\
\hline Até $1000 \mathrm{~g}$ & 1 & $5 \%$ & $5 \%$ & $5 \%$ \\
\hline$\leq 1500 \mathrm{~g}$ & 12 & $60 \%$ & $60 \%$ & $65 \%$ \\
\hline$<2500 \mathrm{~g}$ & 7 & $35 \%$ & $35 \%$ & $100 \%$ \\
\hline TOTAL & 20 & $100 \%$ & $100 \%$ & \\
\hline \multicolumn{5}{|c|}{ Variável 5: Admitidos na UTI } \\
\hline SIM & 10 & $50 \%$ & $50 \%$ & $50 \%$ \\
\hline NÃO & 10 & $50 \%$ & $50 \%$ & $100 \%$ \\
\hline TOTAL & 20 & $100 \%$ & $100 \%$ & \\
\hline \multicolumn{5}{|c|}{ Variável 6: Suporte ventilatório } \\
\hline SIM & 17 & $85 \%$ & $85 \%$ & $85 \%$ \\
\hline TOTAL & $\begin{array}{c}3 \\
20\end{array}$ & $\begin{array}{l}15 \% \\
100 \%\end{array}$ & $\begin{array}{c}15 \% \\
100 \%\end{array}$ & $100 \%$ \\
\hline
\end{tabular}

Fonte: Dados da pesquisa. 
De acordo com os dados do formulário cujos estão apresentados na Tabela 2, 60\% da população do estudo eram do sexo feminino, $60 \%$ nasceram com muito baixo peso, $50 \%$ ficaram internados na UTIN e $85 \%$ necessitaram de suporte ventilatório, fomentando estudos realizados por Rodrigues (2017) o qual diz que o lactente prematuro em sua maioria apresenta baixo peso associado ao nascimento pré-termo e está susceptível a adquirir comorbidade, necessitando assim de internamento na Unidade de Terapia Intensiva Neonatal e Lemos et al. (2010) afirmam que o nascimento antes da 37 semana de IG gera complicações e impactos respiratórios significativos delimitando as principais doenças que acometem o RNPT, tais como, Síndrome do Desconforto Respiratório, Sepse e Icterícia Neonatal associada, necessitando assim de cuidados intensivos.

A Tabela 3 apresenta os dados da GMA, cujas idades corrigidas pós gestacional mais frequentes foram 33 semanas $(n=5)$ e 35 semanas $(n=5)$. Quanto a movimentação geral dos RNPT, 55\% (n=11) apresentaram movimentos anormais do tipo câimbra sincronizada, conforme segue.

Tabela 3. GMA. Vitória da Conquista, Bahia, Brasil. 2021.

\begin{tabular}{|c|c|c|c|c|}
\hline Variáveis & Frequência & Porcentagem & Porcentagem Válida & $\begin{array}{l}\text { Porcentagem } \\
\text { Acumulativa }\end{array}$ \\
\hline \multicolumn{5}{|l|}{ IC Pós Gestacional } \\
\hline $33 \mathrm{~S}$ & 5 & $25,00 \%$ & $25,00 \%$ & $25,00 \%$ \\
\hline $34 \mathrm{~S}$ & 2 & $10,00 \%$ & $10,00 \%$ & $35,00 \%$ \\
\hline $35 \mathrm{~S}$ & 5 & $25,00 \%$ & $25,00 \%$ & $60,00 \%$ \\
\hline $36 \mathrm{~S}$ & 4 & $20,00 \%$ & $20,00 \%$ & $80,00 \%$ \\
\hline $37 \mathrm{~S}$ & 1 & $5,00 \%$ & $5,00 \%$ & $85,00 \%$ \\
\hline $38 \mathrm{~S}$ & 2 & $10,00 \%$ & $10,00 \%$ & $95,00 \%$ \\
\hline $39 \mathrm{~S}$ & 1 & $5,00 \%$ & $5,00 \%$ & $100,00 \%$ \\
\hline TOTAL & 20 & $100,00 \%$ & $100,00 \%$ & \\
\hline Cramped-synchronised GMs (CS) & 11 & $55 \%$ & $55 \%$ & $55 \%$ \\
\hline Normal age-specific GMs & 7 & $35 \%$ & $35 \%$ & $90 \%$ \\
\hline Poor repertoire of GMs (PR) & 2 & $10 \%$ & $10 \%$ & $100 \%$ \\
\hline Chaotic GMs (CH) & 0 & $0 \%$ & $0 \%$ & $100 \%$ \\
\hline TOTAL & 20 & $100 \%$ & $100 \%$ & \\
\hline
\end{tabular}

Fonte: Dados da pesquisa.

A GMA é um instrumento de avaliação rápido e não invasivo baseado na observação e avaliação qualitativa das movimentações espontâneas dos RNs, por meio desse instrumento que avalia a integridade do Sistema Nervoso Central (SNC) é possível identificar bebês com risco de déficits neuromotores (Einspieler, et al., 2016a; Einspieler, et al., 2021). De acordo com Olsen et al. (2018) a GMA quando comparada com outras escalas pode ser utilizada precocemente na avaliação de prematuros, por ser um instrumento observacional, pois não necessita de manuseio e consequentemente não precisa esperar o RNPT estabilizar para tolerar os manejos. Sendo assim, a GMA torna-se bastante efetiva no que tange ao delineamento das intervenções precoces objetivando o desenvolvimento e crescimento adequado do RNPT (Einspieler, et al., 2021).

Os movimentos gerais surgem durante o início da vida fetal, com movimentos lentos e pequenos de flexão lateral da cabeça e / ou tronco, depois aparecem os movimentos lentos, pequenos e simples de braços e pernas (Hadders-algra, 2017). Segundo Einspieler et al. (2016b) são considerados normais os movimentos gerais que tenham variações de sequência, amplitude, velocidade e intensidade e anormais os movimentos que não tenham variabilidade, principalmente na sequência do movimento. 
Pires et al. (2020) trazem que os movimentos são classificados de acordo com a faixa etária: até 40 semanas de IG acontecem os movimentos fetais; de 40 semanas de IG até a $9^{a}$ semana pós termo acontecem os movimentos de contorção (Writhing Movements - WM), caracterizados por serem movimentos de pequena à moderada amplitude, de baixa à moderada velocidade e em uma sequência variável de membros superiores e inferiores, pescoço e tronco; a partir da $9^{\mathrm{a}}$ semana até a $20^{\mathrm{a}}$ semana pós termo tem a presença de movimentos irregulares (Fidgety Movements - FM), caracterizados por serem movimentos de baixa amplitude e velocidade moderada de membros, tronco, cabeça e pequenos movimentos de rotação das mãos e dos pés.

Para Olsen et al. (2018) e Einspieler, et al. (2016b) os movimentos fetais e os movimentos da fase WM são classificados em três categorias: repertório pobre - PR (padrões motores com sequência monótona, pouca variedade e sem complexidade), câimbra sincronizada - CS (movimentos rígidos, sem suavidade e possuem sincronia com movimentos de parar/ iniciar) e caótico - CH (movimentos de grande amplitude e fora dos padrões motores regulares). Os movimentos da fase FM são classificados em normais - FM (movimentos irregulares presentes), ausente - F- (sem movimentos irregulares) e anormal - AF (aumento moderado ou intenso da amplitude e velocidade dos movimentos irregulares) descrito por Pires (2018).

Os RNs avaliados na unidade de cuidados intermediários através da GMA enquadraram-se todos na fase dos Writhing Movements uma vez que apresentaram idades gestacionais corrigidas entre 33 e 39 semanas, destes 55\% apresentaram câimbra sincronizada, $35 \%$ movimentos normais e $10 \%$ pobre repertório, sendo assim, é possível observar que a maioria desses bebês apresentaram movimentos gerais anormais. Os movimentos anormais de câimbra sincronizada são preditivos para paralisia cerebral espástica, já o pobre reportório, são menos preditivos, conforme a literatura de Einspieler et al. (2021).

Olsen et al. (2017) fizeram um estudo com 137 bebês internados no Royal Women's Hospital, Melbourne, Austrália nascidos antes de 30 semanas. Após a seleção dos participantes, os pesquisadores registraram na GMA RNs de 32 semanas de idade corrigida semanalmente e RNs de 34 e 36 semanas quinzenalmente. Os autores encontraram predisposição elevada de movimentos anormais na população analisada. Assim como, os RNs avaliados em nosso estudo apresentaram também em sua maioria movimentos anormais, como a câimbra sincronizada realizando tremor, movimento rígido e simultâneo de MMSS e MMII, e pobre repertório os quais apresentaram diminuição da variação dos movimentos sequenciados.

Estudos apontam que bebês nascidos muito prematuros têm altas chances de apresentarem atrasos no desenvolvimento neuromotor o qual pode repercutir ao longo da vida do indivíduo, porém nem todos esses RNs vão apresentar distúrbio no desenvolvimento (Olsen et al., 2017). Manacero e colaboradores (2012), afirmam que apenas uma gravação dos bebês não é suficiente para afirmar se a alteração do movimento geral, como câimbra sincronizada, vai persistir e ser marcador específico para paralisia cerebral espástica ou se essa alteração do movimento é apenas transitória. Em nosso estudo, foi realizada apenas uma filmagem, pois o tempo de internamento dos bebês, na unidade de cuidados intermediários neonatal, não era prolongado inviabilizando realizar uma nova filmagem e comparar o movimento apresentado na segunda gravação com a avaliação anterior.

\section{Considerações Finais}

Neste estudo foi possível avaliar a movimentação geral dos recém-nascidos prematuros e observar se estavam compatíveis com a idade gestacional ou apresentavam anormalidade nos movimentos, a fim de identificar precocemente alteração no Sistema Nervoso Central e solicitar acompanhamento no serviço de folow-up para intervenções fisioterapêuticas precoces. Ademais evidenciou-se com o desenvolvimento do estudo que o profissional da fisioterapia além de realizar intervenção neurosensoriomotora em recém-nascidos de alto risco para proporcionar o desenvolvimento motor infantil, é capaz de realizar avaliação a fim de detectar sinais clínicos para atrasos de desenvolvimento motor ainda precoce. Toda intervenção 
tem a finalidade de adequar o tônus muscular, diminuir a dor, estimular as sinergias musculares e facilitar o desenvolvimento neurosensóriomotor, percebeu-se no estudo que o instrumento de avaliação GMA, é um facilitador confiável, para avaliação precoce de bebês prematuros.

As limitações do estudo estão relacionadas ao desconforto respiratório apresentados pelos recém-nascidos, bem como, a rotatividade dos bebês internados na unidade, uma vez que recebiam alta hospitalar quando apresentavam estabilidade hemodinâmica e atingiam o peso ideal, inviabilizando a continuidade da segunda fase da avaliação, a fase dos Fidgety Movements.

Por ter sido realizada apenas uma filmagem, pois o tempo de internamento dos bebês na unidade de cuidados intermediários neonatal não era prolongado, sugere-se a realização de novas pesquisas com mais filmagens a fim de comparar o movimento apresentado na segunda gravação com a avaliação anterior.

\section{Referências}

Berger, A. Z et al. (2016). Parto prematuro: características das gestantes de uma população da zona sul de São Paulo. Revista Brasileira de Saúde Materno Infantil, 16(4). https://doi.org/10.1590/1806-93042016000400005.

Bordalo, A. A. (2006). Estudo transversal e/ou longitudinal. Revista Paraense de Medicina, Belém, 20(4). http://scielo.iec.gov.br/scielo.php?script=sci_arttext\&pid=S0101-59072006000400001.

Brasil, M. S. (2016). Guia de Orientações para o Método Canguru na Atenção Básica: Cuidado Compartilhado, Brasília-DF. https://bvsms.saude.gov.br/bvs/publicacoes/guia_orientacoes_metodo_canguru.pdf.

Einspieler, C et al. (2016). The general movement assessment helps us to identify preterm infants at risk for cognitive dysfunction. Frontiers in Psychology.: 406. $10.3389 /$ fpsyg.2016.00406.

Einspieler, C et al. (2016). The general movement optimality score: a detailed assessment of general movements during preterm and term age. Developmental Medicine and Child Neurology, 58(4):361-8. 10.1111/dmcn.12923.

Einspieler, C et al. (2021). The general movement checklist: A guide to the assessment of general movements during preterm and term age. Jornal de Pediatria. 97(4):445-452. 10.1016/j.jped.2020.09.006.

Gil, A.C. (2017). Como elaborar projetos de pesquisa. Atlas.

Gil, A.C. (2010). Como elaborar projetos de pesquisa. Atlas S.A.

Hadders-algra, M. (2017). Neural substrate and clinical significance of general movements: an update. Developmental Medicine and Child Neurology. 60(1):39-46. 10.1111/dmcn.13540.

Lemos, R et al. (2010). Estudo da prevalência de morbidades e complicações neonatais segundo o peso ao nascimento e a idade gestacional em lactentes de um serviço de follow-up. Revista APS, 13(3).: https://periodicos.ufjf.br/index.php/aps/article/view/14411/7779.

Lopes, D. (2017). Evolução nutricional de lactentes nascidos prematuros, acompanhados durante o primeiro ano de vida, no ambulatório de seguimento de um hospital de referência do Rio de Janeiro. Dissertação (Mestrado em Ciências) - FIOCRUZ, <https://www.arca.fiocruz.br/bitstream/icict/24064/2/ve_Daiana_Belen_ENSP_2017.pdf>.

Manacero, S. A et al. (2012). Is it possible to predict the infant's neurodevelopmental outcome at 14 months of age by means of a single preterm assessment of General Movements? Early Human Development. (88). https://doi.org/10.1016/j.earlhumdev.2011.06.013.

Melo, A. M. A. P. A.; Belem, T. O. M. O. U. (2020). Aspectos peculiares do crescimento e desenvolvimento do prematuro. Sociedade de Pediatria de São Paulo. https://www.spsp.org.br/2020/03/09/aspectos-peculiares-do-crescimento-e-desenvolvimento-do-prematuro/.

Olsen, J. E et al. (2018). Preterm and term-equivalent age general movements and 1-year neurodevelopmental outcomes for infants born before 30 weeks' gestation. Developmental Medicine and Child Neurology. 60(1):47-53. 10.1111/dmcn.13558.

Opas. (2018). Quase 30 milhões de recém-nascidos prematuros e doentes necessitam de tratamento para sobreviver todos os anos. <https://www.paho.org/bra/index.php?option=com_content\&view=article\&id=5821:quase-30-milhoes-de-recem-nascidos-prematuros-e-doentes-necessitamde-tratamento-para-sobreviver-todos-os-anos \&Itemid=820>.

OMS. (2018). Nascimento prematuro. <https://www.who.int/en/news-room/fact-sheets/detail/preterm-birth>.

Penha, S. C et al. (2019). Fatores de risco materno associados à prematuridade em uma maternidade-escola. Revista de Políticas Públicas - Sanare, 18(2).: https://sanare.emnuvens.com.br/sanare/article/view/1373.

Pires, C.S et al. (2020). Valor preditivo da general movements assessment na avaliação neurológica de recém-nascidos pré-termo: uma metanálise. Revista Paulista de Pediatria. https://doi.org/10.1590/1984-0462/2020/38/2018286. 
Research, Society and Development, v. 10, n. 15, e257101522781, 2021

(CC BY 4.0) | ISSN 2525-3409 | DOI: http://dx.doi.org/10.33448/rsd-v10i15.22781

Pires, C. S. (2018). Avaliação da general movement assessment em recém-nascidos pré-termo internados em unidade de cuidados intermediários canguru. Dissertação (Mestrado em Ciências), Universidade Estadual de Campinas, Campinas. http://repositorio.unicamp.br/jspui/handle/REPOSIP/333381.

Rodrigues, M. C. (2017). Alimentação e crescimento de lactentes nascidos pré-termo de muito baixo peso egressos de UTI eonatal: Análise de interferência casual, 2017. Dissertação (Mestrado em Saúde Coletiva) - Universidade Federal do Maranhão, http://tedebc.ufma.br:8080/jspui/handle/tede/1312.

Salvagni, K.; Gerzson, L. R.; Almeida, C. S. (2019). Avaliação do desenvolvimento motor de recém-nascidos prematuros extremos e moderados em uma unidade de terapia intensiva neonatal. Revista de Terapia Ocupacional da Universidade de São Paulo, 30(2), 77-85. https://doi.org/10.11606/issn.22386149.v30i2p77-85.

Van Iersel, P et al. (2016). Does general movements quality in term infants predict cerebral palsy and milder forms of limited mobility at 6 years?. Developmental Medicine and Child Neurology. 58(12):1310-1316. https://doi.org/10.1111/dmcn.13228. 